\title{
The mechanism of phenylpyrrole fungicides cannot be explained by a stearic effect upon triososephosphate isomerase alone
}

\author{
Stefan Jacob ${ }^{1}$ and T. Tristan Brandhorst ${ }^{2}$ \\ ${ }^{1}$ Institut für Biotechnologie und Wirkstoff-Forschung gGmbH (IBWF), Erwin-Schrödinger-Str. 56, D-67663 Kaiserslautern \\ ${ }^{2}$ Departments of Pediatrics, School of Medicine and Public Health, University of Wisconsin-Madison, Madison, WI, 53792, USA \\ Arising from T. Tristan Brandhorst et al. 2019, Scientific reports https://doi.org/10.1038/s41598- \\ $\underline{019-41564-9}$
}

The phenylpyrrole fungicide fludioxonil has been used extensively against multiple fungal crop diseases in seed or foliar treatment for over 25 years. To date, very few cases of field resistance have been reported [1] making the phenylpyrrole fungicide class one of the most successful agrochemicals. The mechanism by which fludioxonil affects fungi involves hyperactivation of the HOG signaling pathway through group III hybrid histidine kinases (HHK) [2][3][4]. The mode of action of fludioxonil was never clarified in detail, however. Recent findings speculated that effects upon an upstream target may serve to trigger the group III HHK, resulting in constitutive activation of the HOG pathway [5]. That hyperactivation of the HOG pathway was responsible for fungicidal activity was never in doubt [6][7].

In the respective Scientific Reports article by Brandhorst et al. it was demonstrated that fludioxonil elicited aldehydic and oxidative stress, and that aldehydic stress could mimic the effects of fludioxonil upon fludioxonil sensitive fungi. As triosephosphate isomerase (TPI) can evolve aldehydic stress in the form of methylglyoxal (MGO), and in vitro evidence showed that fludioxonil lead to an elevation of the release of this MGO, it was postulated that TPI may be a target of fludioxonil. Unfortunately, some have interpreted this article to imply that TPI is the sole direct drug target of this fungicide class.

This conclusion is not fully supported by the data, however, as experimental evidence of a direct physical interaction between fludioxonil and TPI in the pathogen has been lacking. All relevant experiments by Brandhorst et al have been conducted in the model yeast, Saccharomyces cerevisiae, which is naturally resistant towards fludioxonil. This yeast was modified to conditionally express a hybrid histidine kinase III 
(i.e. Drk1) known to be essential for fungicidal activity, and thus evidenced fludioxonil sensitivity upon induction of this kinase.

Now, further investigation by the Jacob group clearly demonstrates that TPI cannot be the sole direct target of fludioxonil action. We have specifically inactivated the coding sequence of TPI (MGG_08905) in the genome of the phytopathogenic filamentous ascomycete Magnaporthe oryzae (M. oryzae, anamorph: Pyricularia oryzae). We replaced it with a resistance marker gene and proved the successful deletion of the gene MGG_08905 (TPI) with Southern blot analyses (Fig.S1, supporting material). Were the TPI enzyme the sole direct target of the phenylpyrrole fludioxonil, the null mutants $\Delta M o t p i$ should be resistant to fludioxonil compared to the wildtype strain upon phenylpyrrole treatment. This is not the case.

To explore this, the susceptibility of three independent TPI knockout mutants ( $\triangle M o t p i)$ to the fungicide fludioxonil was examined. Knockouts of the MoHOGl-gene ( $\triangle M o h o g 1$ ) and the M. oryzae HHK MoHIKl-gene (AMohikl) were used as negative controls, since it is established that mutants with an inactivated HOG pathway are resistant towards the phenylpyrrole fludioxonil [4]. Application of fludioxonil resulted in a (lethal) hyperactivation of the HOG pathway in the MoWT, whereas $\triangle M o h o g 1$ and $\Delta M o h i k 1$ were resistant. Although they do not have the TPI enzyme, the $\triangle$ Motpi-mutants $\triangle M o t p i \# 19, \triangle M o t p i \# 25$ and AMotpi\#31 were not significantly less sensitive than MoWT (Fig. 1). Similar results were independently observed in work done by Brandhorst using their fludioxonil-sensitive yeast model (data not shown). 


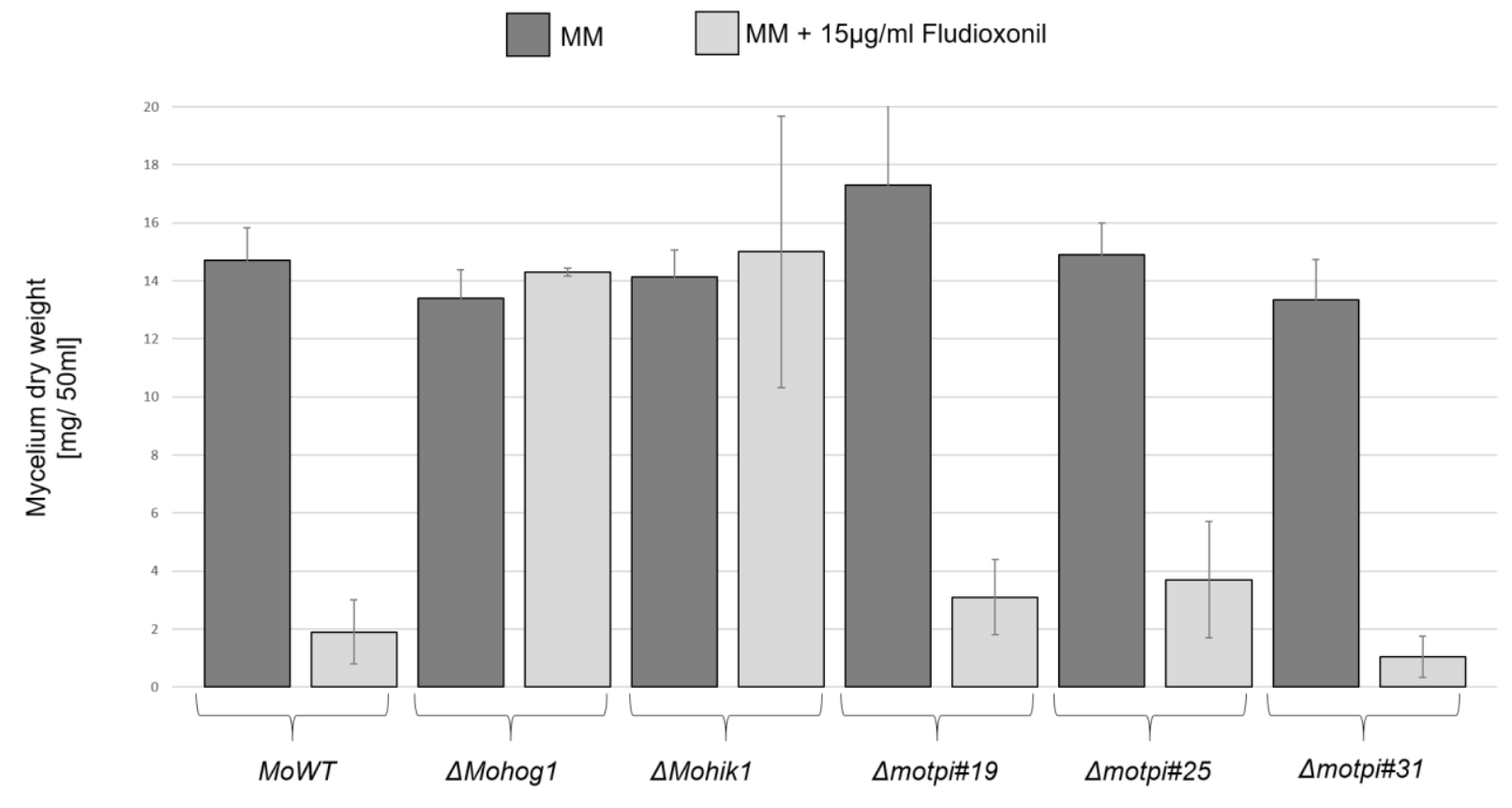

Fig.1: Mycelium dry weight of the Magnaporthe oryzae wildtype strain, the mutants $\Delta M o h o g 1, \Delta M o h i k 1$ and three independent mutants $\Delta M o t p i$ upon fludioxonil treatment. The fungal colonies were grown in MM and in MM including 15 $\mu \mathrm{g} / \mathrm{ml}$ fludioxonil $10 \mathrm{~d}$ at $100 \mathrm{rpm}$ and $26^{\circ} \mathrm{C}$. Error bars represent the standard deviation of three biological replicates of each strain.

Furthermore, the germination ability of conidia after treatment with fludioxonil also was determined for the MoWT and mutant strains $\triangle M o h o g 1, \Delta M o h i k 1$ and the three independent mutants $\triangle M o t p i$. More than $95 \%$ of the MoWT and $\triangle M o t p i$ conidia failed to germinate on medium containing $7.5 \mu \mathrm{g} / \mathrm{ml}$ fludioxonil, whereas the negative control strains, $\triangle M o h o g 1$ and $\triangle M o h i k 1$, exhibited resistance to the fungicide (data not shown).

These results indicate that TPI in filamentous pathogenic fungi is not the sole target of fungicidal action for the phenylpyrrole fludioxonil. Given that the postulated physiological effect of fludioxonil upon TPI involves the evolution of MGO, and the confirmed physiological consequence of a TPI deletion mutation involves significant build-up of cytosolic MGO in mutanted cells [8] (as the MGO precursor, dihydroxyacetone, accumulates during glycolysis), we cannot exclude the possibility that effects upon TPI contribute to a multifactorial mechanism. It might be postulated that the mechanism of fludioxonil toxicity 
involves the induction of both aldehydic and oxidative chemical stressors, and that it is this mixture of stimuli that trigger a response by group III HHKs and, thereafter, the HOG pathway. In the interest of clarity, however, we must specify that the mechanism by which fludioxonil induces its toxic affects cannot depend upon its action on TPI alone.

\section{$\underline{\text { Literature }}$}

[1] Kilani, J. and Fillinger, S. (2016) Phenylpyrroles: 30 years, two molecules and (nearly) no resistance. Frontiers in Microbiology, 7: 2014.

[2] Yoshimi A., Kojima K., Takano Y., and Tanaka, C. (2005) Group III histidine kinase is a positive regulator of Hog1-type mitogen-activated protein kinase in filamentous fungi. Eukaryot Cell 4: 1820e1828.

[3] Tanaka, C., and Izumitsu, K. (2010). Two-component signaling system in filamentous fungi and the mode of action of dicarboximide and phenylpyrrole fungicides. Fungicides, 1, 523-538.

[4] Jacob, S., Foster, A. J., Yemelin, A. and Thines, E. (2015) High osmolarity glycerol (HOG) signalling in Magnaporthe oryzae: Identification of MoYPDl and its role in osmoregulation, fungicide action, and pathogenicity. Fungal Biol. 119, 580-594.

[5] Lawry, S. M., Tebbets, B., Kean, I., Stewart, D., Hetelle, J., and Klein, B. S. (2017). Fludioxonil induces Drk1, a fungal group III hybrid histidine kinase, to dephosphorylate its downstream target, Ypd1. Antimicrobial agents and chemotherapy, 61(2), e01414-16.

[6] Furukawa, K., Randhawa, A., Kaur, H., Mondal, A.K. and Hohmann, S. (2012) Fungal fludioxonil sensitivity is diminished by a constitutively active form of the group III histidine kinase. FEBS Letters, 586: 2417-2422.

[7] Jacob, S., Schüffler, A. and Thines, E. (2016) Hog1p activation by marasmic acid through inhibition of the histidine kinase Sln1p. Pest Management Science, 72: 1268-1274.

[8] Ahmed, N., Battah, S., Karachalias, N., Babaei-jadidi, R., Horanyi, M., Baroti, K., Hollan, S., and Thornalley, P. (2003) Increased formation of methylglyoxal and protein glycation, oxidation and nitrosation in triosephosphate isomerase deficiency. Biochim. et Biophys. Acta (BBA)-Mol. Basis of Disease, 1639(2): 121132.

[9] Odenbach, D., Breth, B., Thines, E., Weber, R. W., Anke, H., and Foster, A. J. (2007). The transcription factor Con7p is a central regulator of infection-related morphogenesis in the rice blast fungus Magnaporthe grisea. Molecular microbiology, 64(2), 293-307.

[10] Bohnert, S., Neumann, H., Thines, E. and Jacob, S. (2019b) Visualizing fungicide action: an in vivo tool for rapid validation of fungicides with target location HOG pathway. Pest Management Science 75(3), 772-778. 


\section{Supporting material}

\section{$\underline{\text { Methods }}$}

\section{$\underline{\text { Strains and culture/growth conditions }}$}

The fungal strain used in this study was Magnaporthe oryzae (M. oryzae 70-15 strain (MoWT), Fungal Genetics Stock Center). The strain was grown at $26^{\circ} \mathrm{C}$ on $\mathrm{CM}(\mathrm{pH} 6.5,2 \%$ agar, containing (per liter) $10 \mathrm{~g}$ glucose, $1 \mathrm{~g}$ yeast extract, $2 \mathrm{~g}$ peptone, $1 \mathrm{~g}$ casamino acids, $50 \mathrm{~mL}$ nitrate salt solution (containing $120 \mathrm{~g}$ $\mathrm{NaNO}_{3}, 10.4 \mathrm{~g} \mathrm{KCl}, 30.4 \mathrm{~g} \mathrm{KH}_{2} \mathrm{PO}_{4}$ and $10.4 \mathrm{~g} \mathrm{MgSO}_{4} \times 7 \mathrm{H}_{2} \mathrm{O}$ per liter) and $1 \mathrm{~mL}$ of a trace element solution (containing $22 \mathrm{~g} \mathrm{ZnSO}_{4} \times 7 \mathrm{H}_{2} \mathrm{O}, 11 \mathrm{~g} \mathrm{H}_{3} \mathrm{BO}_{3}, 5 \mathrm{~g} \mathrm{MnCl}_{2}$ × $4 \mathrm{H}_{2} \mathrm{O}, 5 \mathrm{~g} \mathrm{FeSO} 4$ x $7 \mathrm{H}_{2} \mathrm{O}, 1.7 \mathrm{~g}$ $\mathrm{CoCl}_{2}$ x $6 \mathrm{H}_{2} \mathrm{O}, 1.6 \mathrm{~g} \mathrm{CuSO}_{4}$ x $5 \mathrm{H}_{2} \mathrm{O}, 1.5 \mathrm{~g} \mathrm{Na}_{2} \mathrm{MoO}_{4}$ x $2 \mathrm{H}_{2} \mathrm{O}$ and $50 \mathrm{~g} \mathrm{Na} 2 \mathrm{EDTA}$, pH 6.5 adjusted by $1 \mathrm{M}$ $\mathrm{KOH}$ per liter). Minimal medium (pH 6.5) contains $1 \mathrm{~g}$ glucose, $0.25 \mathrm{~mL}$ of a $0.01 \%$ biotin solution, $50 \mathrm{~mL}$ nitrate salt solution, $1 \mathrm{~mL}$ of a trace element solution and $1 \mathrm{~mL}$ of a $1 \%$ thiamine dichloride solution per liter.

DNA manipulations and construction of fungal transformation vectors

The DNA of Magnaporthe oryzae was isolated from mycelia of 3 day old liquid cultures (grown in CM at $26^{\circ} \mathrm{C}$ and $100 \mathrm{rpm}$ ) using the DNeasy® plant mini Kit (Quiagen $\mathrm{GmbH}$, Hilden), following the manufacturer's instructions for purification of DNA from plants and filamentous fungi. The DNA manipulation followed standard procedures. Escherichia coli XL1-BLUE strain (Stratagene) or NEB® 10ß Competent Escherichia coli strains (High Efficiency) were used for routine bacterial transformations and the construction of plasmids. Fungal transformation of M. oryzae was conducted using Agrobacterium tumefaciens-mediated transformation (ATMT). The detailed procedures followed those described previously [9].

The coding gene sequence of MoTPI (MGG_08905) was completely replaced by the HPT gene from pSJ+GFP [10], using the Gibson Assembly ${ }^{\circledR}$ cloning method, for the generation of the "lof" mutant $\Delta$ Motpi. The flanking regions (1000 bp) of MoTPI were amplified by PCR from genomic DNA with primers MoTPIfl-for: ATATATTGTGGTGTAAACAACGTCAGCAGTCCTATAATGATGAGCC, MotPI-fl-rev: 
TAACGTCGACAAGAATGTGTCGCGTTCGTCTGGTG, TAACGTCGACAGTATGGTTGGTTGACGTTTTGCGG
MoTPI-f2-for:

and
MoTPI-f2-rev:

ttctcttaggtttacctgcaaagaggttgaattgtagtgtcaagttgtagag. The HPT gene was amplified from pSJ+GFP using the primer MoTPI-HPT-for: ACACATTCTTGTCGACGTTAACTGATATTGAAGGAGCATTTTTTG and MoTPI-HPT-rev: CAACCATACTGTCGACGTTAACTGGTTCCCGGTCG. The backbone vector was an PstI/BglII-restricted pSJ-basic. The resulting 7917 bp plasmid pSJ-TPI-HPT was used to transform MoWT via ATMT, resulting in the mutant strain $\Delta M o t p i$.

\section{$\underline{\text { Antifungal activity assays }}$}

Antifungal activity of germinating conidia was tested in accordance with [7]. Conidia were harvested from eleven-day-old M. oryzae cultures and the mutant strains and grown on CM. The conidia were filtered through two layers of miracloth tissue (Merck) to give a conidial suspension, which was adjusted to $5 \times 10^{4}$ conidia/mL in $\mathrm{H}_{2} \mathrm{O}$. The test compounds were then added and the samples were incubated at $26{ }^{\circ} \mathrm{C}$ for at least $16 \mathrm{~h}$. The germination, i.e. the subsequent initial vegetative growth phase, was monitored under the microscope. The values were calculated by counting the number of germinated conidia out of 100 for each sample.

We determined the mycelium dry weight of the fungal colonies grown in liquid culture to determine the antifungal activity of fludioxonil on vegetative growth. The cultures were grown in liquid MM and on MM including $15 \mu \mathrm{g} \mathrm{ml}^{-1}$ fludioxonil for $10 \mathrm{~d}$ at $100 \mathrm{rpm}$ and $26^{\circ} \mathrm{C}$.

\section{Figures}

Fig.S1: Southern blot analysis of gene inactivation mutants in $M$. oryzae with specific probes. Genomic DNA of $M$. oryzae strain 70-15 and the TPI (MGG_08905)-mutants were isolated and restricted with HindIII restriction enzymes. The probes which we used for hybridization with the genomic DNA of the wild-type strain and the corresponding mutant strains were always identical. The pink marked primer pairs marked the position of the hybridization of the probes in the schematic presentation of the genomic DNA. 


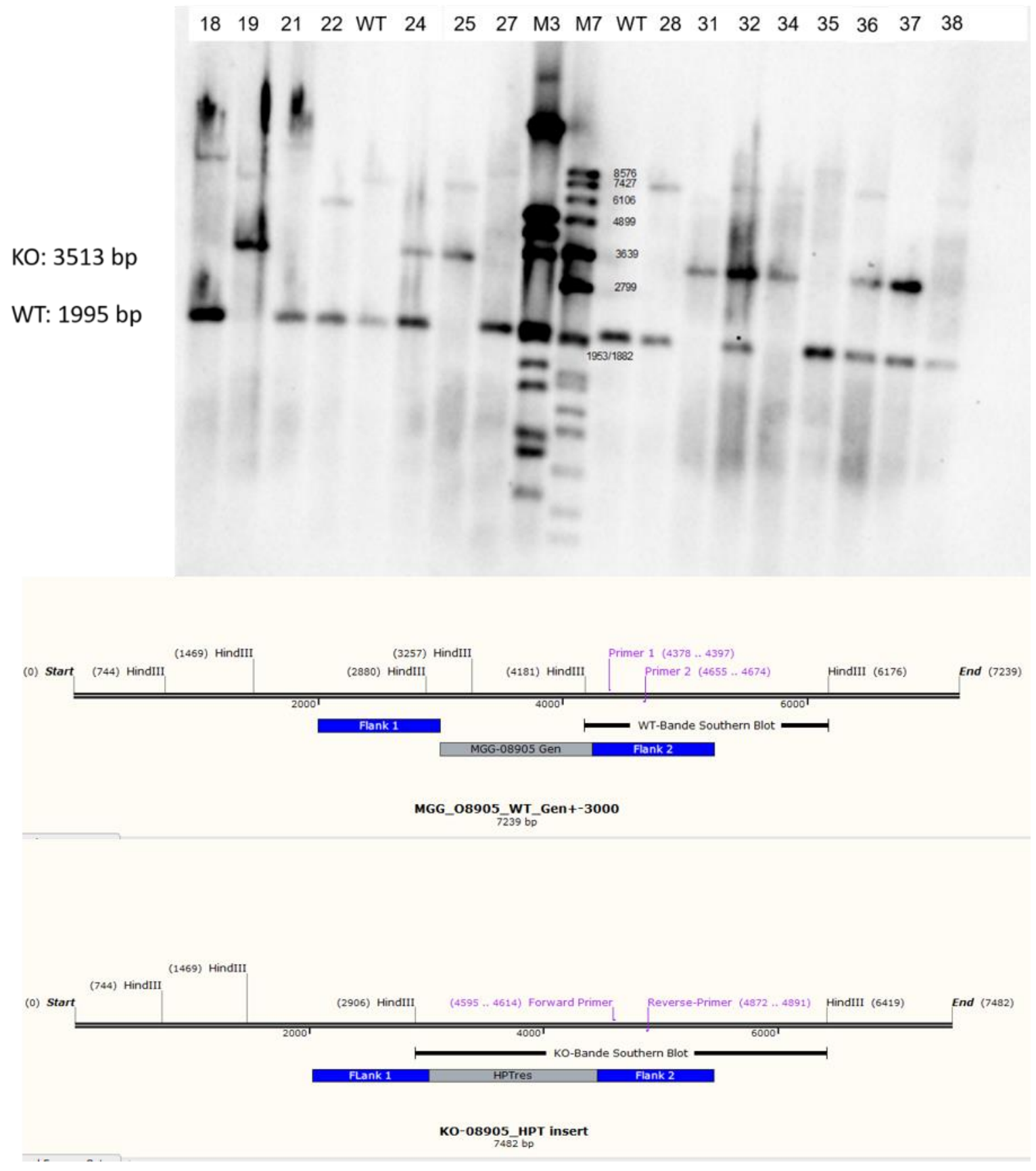

